MPP-2009-42

LMU-ASC 18/09

\title{
LHC Phenomenology for String Hunters
}

\author{
Luis A. Anchordoqui, ${ }^{1}$ Haim Goldberg, ${ }^{2}$ Dieter Lüst, ${ }^{3,4}$ \\ Satoshi Nawata, ${ }^{1,3}$ Stephan Stieberger, ${ }^{3}$ and Tomasz R. Taylor ${ }^{2}$ \\ ${ }^{1}$ Department of Physics, \\ University of Wisconsin-Milwaukee, Milwaukee, WI 53201, USA \\ ${ }^{2}$ Department of Physics, \\ Northeastern University, Boston, MA 02115, USA \\ ${ }^{3}$ Max-Planck-Institut für Physik \\ Werner-Heisenberg-Institut, 80805 München, Germany \\ ${ }^{4}$ Arnold Sommerfeld Center for Theoretical Physics \\ Ludwig-Maximilians-Universität München, 80333 München, Germany
}

(Dated: April 2009)

\begin{abstract}
We consider extensions of the standard model based on open strings ending on D-branes, with gauge bosons due to strings attached to stacks of D-branes and chiral matter due to strings stretching between intersecting D-branes. Assuming that the fundamental string mass scale is in the $\mathrm{TeV}$ range and the theory is weakly coupled, we discuss possible signals of string physics at the Large Hadron Collider (LHC). In previous works, direct channel excitations of Regge recurrences in parton-parton scattering supplied the outstanding new signature. The present work considers the deviation from standard model expectations for the 4-fermion processes $q q \rightarrow q q$ and $q q^{\prime} \rightarrow q q^{\prime}$, in which the $s$-channel excitation of string resonances is absent. In this case, we find that KaluzaKlein recurrences at masses somewhat less than the string scale generate effective 4-fermion contact terms which can significantly enhance the dijet $R$ ratio above its QCD value of about 0.6. The simultaneous observation of a nearby resonant structure in the dijet mass spectrum would provide a "smoking gun" for TeV scale string theory. In this work, we also show that (1) for $M_{\text {string }}<3.5 \mathrm{TeV}$, the rates for various topologies arising from the $p p \rightarrow Z^{0}+$ jet channel could deviate significantly from standard model predictions and (2) that the sizeable cross sections for Regge recurrences can allow a $6 \sigma$ discovery for string scales as large as $3 \mathrm{TeV}$ after about 1 year of LHC operation at $\sqrt{s}=10 \mathrm{TeV}$ and $\int \mathcal{L} d t \sim 100 \mathrm{pb}^{-1}$.
\end{abstract}




\section{INTRODUCTION}

Two of the most important scales in physics are the Planck scale $\left(M_{\mathrm{Pl}}=G_{\mathrm{N}}^{-1 / 2} \approx\right.$ $\left.10^{19} \mathrm{GeV}\right)$ and the weak scale $\left(M_{\mathrm{W}} \approx G_{\mathrm{F}}^{-1 / 2} \approx 300 \mathrm{GeV}\right)$, and there is a longstanding problem in explaining the disparity between these scales. The traditional view is to adopt $M_{\mathrm{Pl}}$ as the fundamental scale and attempt to derive $M_{\mathrm{W}}$ through some dynamical mechanism (e.g. renormalization group evolution). In recent years, however, a new framework with a diametrically opposite viewpoint has been proposed, in which $M_{\mathrm{W}}$ is instead the fundamental scale of nature [1]. D-brane string compactifications with low string scale and large extra dimensions allow a definite representation of this innovative premise [2].

TeV-scale superstring theory provides a brane-world description of the standard model (SM), which is localized on membranes extending in $p+3$ spatial dimensions, the so-called D-branes. Gauge interactions emerge as excitations of open strings with endpoints attached on the D-branes, whereas gravitational interactions are described by closed strings that can propagate in all nine spatial dimensions of string theory (these comprise parallel dimensions extended along the $(p+3)$-branes and transverse dimensions). The apparent weakness of gravity at energies below few TeVs can then be understood as a consequence of the gravitational force "leaking" into the transverse compact dimensions of spacetime. This is possible only if the intrinsic scale of string excitations is also of order a few TeVs. Should nature be so cooperative, a whole tower of infinite string excitations will open up at this low mass threshold, and new particles of spin $j$ follow the well known Regge trajectories of vibrating strings: $j=j_{0}+\alpha^{\prime} M^{2}$, where $\alpha^{\prime}$ is the Regge slope parameter that determines the fundamental string mass scale

$$
M_{s}=\frac{1}{\sqrt{\alpha^{\prime}}} .
$$

In a series of recent publications [3-7] we have computed open string scattering amplitudes in D-brane models and have discussed ${ }^{1}$ the associated phenomenological aspects of low mass string Regge recurrences related to experimental searches for physics beyond the SM at the LHC. In this work we extend our previous discussion in three directions. The first is a calculation of non-resonant $t$ - and $u$-channel exchanges for the dominant $q q \rightarrow q q$ subprocess contributing to hadronic dijets. The second is a calculation of stringy contributions to $p p \rightarrow$ monojet, $p p \rightarrow$ dilepton + jet, and $p p \rightarrow$ trijet, which parallels our previous analysis of $p p \rightarrow \gamma+$ jet $[3,4]$. The third is an update of our dijet analysis to asses the range of discovery of stringy resonances in the first run of the LHC at $10 \mathrm{TeV}$ center-of-mass energy.

We consider the extensions of the SM based on open strings ending on D-branes, with gauge bosons due to strings attached to stacks of D-branes and chiral matter due to strings stretching between intersecting D-branes [12]. To develop our program in the simplest way, we will work within the construct of a minimal model in which we consider scattering processes which take place on the (color) $U(3)_{a}$ stack of D-branes, which is intersected by the (weak doublet) $U(2)_{b}$ stack of D-branes, as well by a third (weak singlet) $U(1)_{c}$ stack of D-brane. These three stacks of $\mathrm{D}(3+\mathrm{p})$-branes entirely fill the uncompactified part of space-time and wrap certain $p$-cycles $\Sigma^{(a, b, c)}$ inside the compact six-dimensional manifold $M_{6}$. In the bosonic sector, the open strings terminating on the $U(3)_{a}$ stack contain the

\footnotetext{
${ }^{1}$ String Regge resonances in models with low string scale are also discussed in [8, 9], while KK graviton exchange into the bulk, which appears at the next order in perturbation theory, is discussed in $[8,10,11]$.
} 
standard gluons $g$ and an additional $U(1)_{a}$ gauge boson $C$; on the $U(2)_{b}$ stacks the open strings correspond to the weak gauge bosons $W$, and again an additional $U(1)_{b}$ gauge field. So the associated gauge groups for these stacks are $S U(3)_{\mathrm{C}} \times U(1)_{a}, S U(2)_{\mathrm{EW}} \times U(1)_{b}$, and $U(1)_{c}$, respectively; the physical hypercharge is a linear combination of $U(1)_{a}, U(1)_{b}$, and $U(1)_{c}$, plus in general a forth $U(1)_{d}$, which is not relevant for the following discussion. The fermionic matter consists of open strings, which stretch between different stacks of $\mathrm{D}(p+3)$ branes and are hence located at the intersection points. Concretely, the left-handed quarks are sitting at the intersection of the $a$ and the $b$ stacks, whereas the right-handed $u$ quarks comes from the intersection of the $a$ and $c$ stacks and the right-handed $d$ quarks are situated at the intersection of the $a$ stack with the $c^{\prime}$ (orientifold mirror) stack. All the scattering amplitudes between these SM particles, which we will need in the following, essentially only depend on the local intersection properties of these D-brane stacks.

Such intersecting D-brane models involve at least three kinds of generic mass scales. First, of course, there is the fundamental string scale, given in (1) in terms of the slope parameter $\alpha^{\prime}$. Second, compactification from ten to four dimensions on an internal six-dimensional space of Volume $V_{6}$ defines a mass scale:

$$
M_{6}=\frac{1}{V_{6}^{1 / 6}} .
$$

Third, wrapping the color stack $a$ and the weak stack $b$ of $\mathrm{D}(p+3)$-branes (we are neglecting the other stacks for the moment) around the internal $p$-cycles $\Sigma^{(a, b)}$ defines two volumes $V_{p}^{(a, b)}$ of these sub-spaces and their associated masses $\left(V_{p}^{(a, b)}=(2 \pi)^{p} v_{p}^{(a, b)}\right)$ :

$$
M_{p}^{(a, b)}=\frac{1}{\left(v_{p}^{(a, b)}\right)^{1 / p}} .
$$

In order to streamline our discussion of Kaluza-Klein and string winding modes associated to internal $p$-cycles we make a simplifying assumption that they are "isotropic" i.e. one needs only one scale to characterize each $p$-cycle.

These three types of fundamental dimensional parameters of D-brane models are linked to 4D physical observables in the following way: First, the 4D Planck mass given by

$$
M_{\mathrm{Pl}}^{2}=8 e^{-2 \phi_{10}} M_{s}^{8} \frac{V_{6}}{(2 \pi)^{6}}
$$

determines the strength of gravitational interactions. Here, the dilaton field $\phi_{10}$ is related to the string coupling constant through $g_{s}=e^{\phi_{10}}$. Thus, for a string scale $M_{s} \approx \mathcal{O}(1 \mathrm{TeV})$, the volume of the internal space $M_{6}$ needs to be as large as $V_{6} M_{s}^{6}=\mathcal{O}\left(10^{32}\right)$. Second, the $4 \mathrm{D}$ gauge couplings of the strong and weak interactions are given in terms of the volumes $V_{p}^{(a, b)}$ as

$$
g_{(a, b)}^{-2}=(2 \pi)^{-1} M_{s}^{p} e^{-\phi_{10}} v_{p}^{(a, b)} .
$$

Again for a string scale $M_{s} \approx \mathcal{O}(1 \mathrm{TeV})$ and using the known values of the strong and weak gauge coupling constants: $g_{a}^{2} / 4 \pi \equiv g_{3}^{2} / 4 \pi \approx 0.1$ and $g_{b}^{2} / 4 \pi \equiv g_{2}^{2} / 4 \pi \approx \frac{1}{3} g_{3}^{2} / 4 \pi$ at the string scale $\left(g_{2}^{2} / 4 \pi=\alpha_{\mathrm{em}} / \sin ^{2} \theta_{W}, \sin ^{2} \theta_{W} \approx 0.23, \alpha_{\mathrm{em}} \approx 1 / 128\right)$ we can compute the volumes of the internal cycles, assuming weak string coupling. To be specific, we choose $g_{s}=0.2$, and then we obtain:

$$
M_{s}^{p} v_{p}^{(a)} \approx 1, \quad M_{s}^{p} v_{p}^{(b)} \approx 3 .
$$


The string mass scale $M_{s}$ and the volumes $V_{p}^{(a, b)}$ of the internal cycles are closely related to the masses of those stringy particles that can be possibly seen at the LHC. First, there is the infinite tower of open string Regge excitations of the known SM particles. These are completely model independent, i.e. independent of the compactified dimensions. They have the same quantum numbers under the SM gauge group as the gluons and the quarks, but in general higher spins, and their masses are just square-root-of-integer multiples of the string mass $M_{s}$. The first Regge excitations of the gluon $(g)$ and quarks $(q)$ will be denoted by $g^{*}, q^{*}$, respectively. The first excitation of the $C$ (the extra $U(1)$ boson tied to the color stack) will be denoted by $C^{*}$. Only one assumption will be necessary in order to set up a solid framework: the string coupling must be small for the validity of perturbation theory in the computations of scattering amplitudes. In this case, black hole production and other strong gravity effects occur at energies above the string scale, therefore at least the few lowest Regge recurrences are available for examination, free from interference with some complex quantum gravitational phenomena. In fact, as discussed in Refs. [3-7], some basic properties of Regge resonances like their production rates and decay widths are completely model-independent.

Second, in any D-brane compactification there is an infinite tower of internal open string KK excitations of the SM fields along the $\mathrm{D}(p+3)$-branes. These particles have SM quantum numbers and, if the internal cycles are isotropic, their masses can be written as

$$
M_{K K}^{(a, b)}=\frac{n}{\left(v_{p}^{(a, b)}\right)^{1 / p}},
$$

where the integer $n$ labels individual KK mass levels. Note that these levels may be degenerate: for instance, the lowest KK level is typically $p$-fold degenerate in orientifold compactifications. Using the same value for the string coupling constant we can, using Eqs.(6) and (7), compute the masses of the first $(n=1)$ strong and weak KK excitations for two possible brane scenarios, namely IIA orientifold with intersecting D6-branes $(p=3)$ and IIB orientifolds with wrapped D7-branes $(p=4)$ :

$$
\begin{array}{ll}
p=3: & M_{K K}^{(a)} \equiv M_{K K}^{(3)} \approx M_{s}, \quad M_{K K}^{(b)} \equiv M_{K K}^{(2)} \approx 0.70 M_{s}, \\
p=4: & M_{K K}^{(a)} \equiv M_{K K}^{(3)} \approx M_{s}, \quad M_{K K}^{(b)} \equiv M_{K K}^{(2)} \approx 0.75 M_{s} .
\end{array}
$$

The presence of these KK modes is a generic property of D-brane models however their masses and their couplings to the SM particles depend on the details of the compactification manifold. Depending on the internal topology, there is also the possibility of charged winding states around internal 1-cycles inside $\Sigma_{a, b, c}$. If they exist, e.g. in toroidal-like compactifications, their masses are proportional to the radii of the wrapped cycles:

$$
M_{\text {wind. }}^{(a, b)}=m\left(v_{p}^{(a, b)}\right)^{1 / p} M_{s}^{2} .
$$

Typically the parameter $m$ is an integer and accounts for winding numbers. More generally, $m$ may also be a positive real number comprising further details of the $\mathrm{D}$-brane setup like intersection angles or displacement. Here again, one expects a certain degeneracy in each level.

Finally, in addition to the (universal) Regge, KK and winding modes, which are charged under the SM, there typically exist several other neutral particles, like transverse KK modes, several moduli scalars and other closed string states. However, these states interact only 
gravitationally, i.e. at string one-loop, with the SM fields, and hence are not relevant for LHC physics.

The layout of the paper is as follows. Section II presents an overview of all formulae relevant for the $s^{-}, t-$, and $u$-channel string amplitudes of lowest massive Regge and KaluzaKlein (KK) excitations leading to dijets. Armed with the full-fledged string amplitudes of all partonic subprocesses, in Sec. III we study the main features of the dijet angular distributions and we elucidate how future measurements of these distributions can provide a potent method for distinguishing between different compactification scenarios. In Sec. IV we study the various final-state topologies arising in the $p p \rightarrow Z^{0}+$ jet channel. We show that, for $M_{s}<4 \mathrm{TeV}$, monojet, trijet, and dilepton plus jet configurations could provide corroborative evidence for Regge excitations in D-brane models. In Sec. V we quantify signal and background rates of Regge recurrences in the early LHC run, operating at $5 \mathrm{TeV}$ per beam. We show that dijet signals of string excitations at the LHC may be so large that they could be exposed to in the early days of running. We summarize our conclusions in Sec. VI.

\section{DIJET SCATTERING AMPLITUDES}

\section{A. Regge recurrences}

The physical processes underlying dijet production in $p p$ and $p \bar{p}$ collisions are the scattering of two partons $i j$, producing two final partons $k l$ that fragment into hadronic jets. The corresponding $2 \rightarrow 2$ scattering amplitudes $\mathcal{M}(i j \rightarrow k l)$, computed at the leading order in string perturbation theory, are collected in Ref. [6]. The amplitudes involving four gluons as well as those with two gluons plus two quarks do not depend on the compactification details and are completely model-independent. All string effects are encapsulated in these amplitudes in one "form factor" function of Mandelstam variables $s, t, u$ (constrained by $s+t+u=0)$ :

$$
V(s, t, u)=\frac{s u}{t M_{s}^{2}} B\left(-s / M_{s}^{2},-u / M_{s}^{2}\right)=\frac{\Gamma\left(1-s / M_{s}^{2}\right) \Gamma\left(1-u / M_{s}^{2}\right)}{\Gamma\left(1+t / M_{s}^{2}\right)} .
$$

The physical content of the form factor becomes clear after using the well-known expansion in terms of $s$-channel resonances [13]:

$$
B\left(-s / M_{s}^{2},-u / M_{s}^{2}\right)=-\sum_{n=0}^{\infty} \frac{M_{s}^{2-2 n}}{n !} \frac{1}{s-n M_{s}^{2}}\left[\prod_{J=1}^{n}\left(u+M_{s}^{2} J\right)\right],
$$

which exhibits $s$-channel poles associated to the propagation of virtual Regge excitations with masses $\sqrt{n} M_{s}$. Thus near the $n$th level pole $\left(s \rightarrow n M_{s}^{2}\right)$ :

$$
V(s, t, u) \approx \frac{1}{s-n M_{s}^{2}} \times \frac{M_{s}^{2-2 n}}{(n-1) !} \prod_{J=0}^{n-1}\left(u+M_{s}^{2} J\right) .
$$

In specific amplitudes, the residues combine with the remaining kinematic factors, reflecting the spin content of particles exchanged in the $s$-channel, ranging from $J=0$ to $J=n+1$.

In the following we isolate the contribution to the partonic cross section from the first resonant state. The $s$-channel pole terms of the average square amplitudes contributing to 
dijet production at the LHC can be obtained from the general formulae given in Ref. [6], using Eq.(12). However, for phenomenological purposes, the poles need to be softened to a Breit-Wigner form by obtaining and utilizing the correct total widths of the resonances [5]. After this is done, the contributions of the various channels are as follows [7]:

$$
\begin{aligned}
& |\mathcal{M}(g g \rightarrow g g)|^{2}=\frac{19}{12} \frac{g^{4}}{M_{s}^{4}}\left\{W_{g^{*}}^{g g \rightarrow g g}\left[\frac{M_{s}^{8}}{\left(s-M_{s}^{2}\right)^{2}+\left(\Gamma_{g^{*}}^{J=0} M_{s}\right)^{2}}+\frac{t^{4}+u^{4}}{\left(s-M_{s}^{2}\right)^{2}+\left(\Gamma_{g^{*}}^{J=2} M_{s}\right)^{2}}\right]\right. \\
& \left.+W_{C^{*}}^{g g \rightarrow g g}\left[\frac{M_{s}^{8}}{\left(s-M_{s}^{2}\right)^{2}+\left(\Gamma_{C^{*}}^{J=0} M_{s}\right)^{2}}+\frac{t^{4}+u^{4}}{\left(s-M_{s}^{2}\right)^{2}+\left(\Gamma_{C^{*}}^{J=2} M_{s}\right)^{2}}\right]\right\} \text {, } \\
& |\mathcal{M}(g g \rightarrow q \bar{q})|^{2}=\frac{7}{24} \frac{g^{4}}{M_{s}^{4}} N_{f} \quad\left[W_{g^{*}}^{g g \rightarrow q \bar{q}} \frac{u t\left(u^{2}+t^{2}\right)}{\left(s-M_{s}^{2}\right)^{2}+\left(\Gamma_{g^{*}}^{J=2} M_{s}\right)^{2}}\right. \\
& \left.+W_{C^{*}}^{g g \rightarrow q \bar{q}} \frac{u t\left(u^{2}+t^{2}\right)}{\left(s-M_{s}^{2}\right)^{2}+\left(\Gamma_{C^{*}}^{J \bar{N}^{2}} M_{s}\right)^{2}}\right] \\
& |\mathcal{M}(q \bar{q} \rightarrow g g)|^{2}=\frac{56}{27} \frac{g^{4}}{M_{s}^{4}}\left[W_{g^{*}}^{q \bar{q} \rightarrow g g} \frac{u t\left(u^{2}+t^{2}\right)}{\left(s-M_{s}^{2}\right)^{2}+\left(\Gamma_{g^{*}}^{J=2} M_{s}\right)^{2}}\right. \\
& \left.+W_{C^{*}}^{q \bar{q} \rightarrow g g} \frac{u t\left(u^{2}+t^{2}\right)}{\left(s-M_{s}^{2}\right)^{2}+\left(\Gamma_{C^{*}}^{J=2} M_{s}\right)^{2}}\right] \text {, } \\
& |\mathcal{M}(q g \rightarrow q g)|^{2}=-\frac{4}{9} \frac{g^{4}}{M_{s}^{2}}\left[\frac{M_{s}^{4} u}{\left(s-M_{s}^{2}\right)^{2}+\left(\Gamma_{q^{*}}^{J=1 / 2} M_{s}\right)^{2}}+\frac{u^{3}}{\left(s-M_{s}^{2}\right)^{2}+\left(\Gamma_{q^{*}}^{J=3 / 2} M_{s}\right)^{2}}\right],
\end{aligned}
$$

where $g$ is the QCD coupling constant $\left(\alpha_{\mathrm{QCD}}=\frac{g^{2}}{4 \pi} \approx 0.1\right)$ and $\Gamma_{g^{*}}^{J=0}=75\left(M_{s} / \mathrm{TeV}\right) \mathrm{GeV}$, $\Gamma_{C^{*}}^{J=0}=150\left(M_{s} / \mathrm{TeV}\right) \mathrm{GeV}, \Gamma_{g^{*}}^{J=2}=45\left(M_{s} / \mathrm{TeV}\right) \mathrm{GeV}, \Gamma_{C^{*}}^{J=2}=75\left(M_{s} / \mathrm{TeV}\right) \mathrm{GeV}, \Gamma_{q^{*}}^{J=1 / 2}=$ $\Gamma_{q^{*}}^{J=3 / 2}=37\left(M_{s} / \mathrm{TeV}\right) \mathrm{GeV}$ are the total decay widths for intermediate states $g^{*}, C^{*}$, and $q^{*}$ (with angular momentum $J$ ) [5]. The associated weights of these intermediate states are given in terms of the probabilities for the various entrance and exit channels

$$
\begin{gathered}
W_{g^{*}}^{g g \rightarrow g g}=\frac{8\left(\Gamma_{g^{*} \rightarrow g g}\right)^{2}}{8\left(\Gamma_{g^{*} \rightarrow g g}\right)^{2}+\left(\Gamma_{C^{*} \rightarrow g g}\right)^{2}}=0.44, \\
W_{C^{*}}^{g g \rightarrow g g}=\frac{\left(\Gamma_{C^{*} \rightarrow g g}\right)^{2}}{8\left(\Gamma_{g^{*} \rightarrow g g}\right)^{2}+\left(\Gamma_{C^{*} \rightarrow g g}\right)^{2}}=0.56, \\
W_{g^{*}}^{g g \rightarrow q \bar{q}}=W_{g^{*}}^{q \bar{q} \rightarrow g g}=\frac{8 \Gamma_{g^{*} \rightarrow g g} \Gamma_{g^{*} \rightarrow q \bar{q}}}{8 \Gamma_{g^{*} \rightarrow g g} \Gamma_{g^{*} \rightarrow q \bar{q}}+\Gamma_{C^{*} \rightarrow g g} \Gamma_{C^{*} \rightarrow q \bar{q}}}=0.71, \\
W_{C^{*}}^{g g \rightarrow q \bar{q}}=W_{C^{*}}^{q \bar{q} \rightarrow g g}=\frac{\Gamma_{C^{*} \rightarrow g g} \Gamma_{C^{*} \rightarrow q \bar{q}}}{8 \Gamma_{g^{*} \rightarrow g g} \Gamma_{g^{*} \rightarrow q \bar{q}}+\Gamma_{C^{*} \rightarrow g g} \Gamma_{C^{*} \rightarrow q \bar{q}}}=0.29 .
\end{gathered}
$$

Superscripts $J=2$ are understood to be inserted on all the $\Gamma$ 's in Eqs.(17), (18), (19), (20). Equation (13) reflects the fact that weights for $J=0$ and $J=2$ are the same [5]. In what follows we set the number of flavors $N_{f}=6$. 


\section{B. KK and winding modes}

The amplitudes for the four-fermion processes like quark-antiquark scattering are more complicated because the respective form factors describe not only the exchanges of Regge states but also of heavy Kaluza-Klein and winding states with a model-dependent spectrum determined by the geometry of extra dimensions [14]. Consider e.g. the quark-quark scattering process. The square amplitude for the identical quark flavors $q q \rightarrow q q$ is given by $[6]$

$$
\begin{aligned}
|\mathcal{M}(q q \rightarrow q q)|^{2} & =g^{4}\left\{\frac{2}{9} \frac{1}{t^{2}}\left[\left(s F_{t u}^{b b}\right)^{2}+\left(s F_{t u}^{c c}\right)^{2}+\left(u G_{t u}^{b c}\right)^{2}+\left(u G_{t u}^{\prime c b}\right)^{2}\right]\right. \\
& +\frac{2}{9} \frac{1}{u^{2}}\left[\left(s F_{u t}^{b b}\right)^{2}+\left(s F_{u t}^{c c}\right)^{2}+\left(t G_{u t}^{\prime b c}\right)^{2}+\left(t G_{u t}^{\prime c b}\right)^{2}\right] \\
& \left.-\frac{4}{27} \frac{s^{2}}{t u}\left(F_{t u}^{b b} F_{u t}^{b b}+F_{t u}^{c c} F_{u t}^{c c}\right)\right\}
\end{aligned}
$$

whereas for different flavors $q q^{\prime} \rightarrow q q^{\prime}$

$$
\left|\mathcal{M}\left(q q^{\prime} \rightarrow q q^{\prime}\right)\right|^{2}=g^{4} \frac{2}{9} \frac{1}{t^{2}}\left[\left(s F_{t u}^{b b}\right)^{2}+\left(s G_{t u}^{c c^{\prime}}\right)^{2}+\left(u G_{t u}^{\prime b c}\right)^{2}+\left(u G_{t u}^{\prime b c^{\prime}}\right)^{2}\right]
$$

where

$$
\begin{aligned}
F_{t u}^{b b} & =\frac{t}{g^{2}} V_{a b a b}\left(-t / M_{s}^{2},-u / M_{s}^{2}\right) \\
G_{t u}^{b c} & =\frac{t}{g^{2}} V_{a b a c}\left(-t / M_{s}^{2},-u / M_{s}^{2}\right) \\
G_{t u}^{\prime b c} & =\frac{t}{g^{2}} V_{a b a c}^{\prime}\left(-t / M_{s}^{2},-u / M_{s}^{2}\right),
\end{aligned}
$$

with the $V$-functions on the r.h.s. defined in Eq.(5.71) of Ref. [6]. Note that the above definitions single out the QCD coupling constant $g \equiv g_{a}$, although the $V$-functions depend on the geometry of all internal cycles associated D-branes. For example,

$$
V_{a b a b}(t, u)=2 \pi \alpha^{\prime} g_{s} \int_{0}^{1} d x x^{t-1}(1-x)^{u-1} I(x) \sum_{p_{a}, p_{b} \in \mathbb{Z}} e^{-S_{\text {inst. }}^{b a}(x)},
$$

where $I(x)$ is the "quantum" part of the four-fermion string amplitude while the exponential $e^{-S_{\text {inst. }}^{b a}(x)}$ weights the instanton contributions of D-branes wrapping various internal cycles, with $p_{a}$ and $p_{b}$ counting the winding numbers of $a$ - and $b$-stacks along the respective cycles. The function $V_{a b a b}(t, u)$ has kinematic poles in $t$ - and $u$-channels, originating from $x \rightarrow$ 0 and $x \rightarrow 1$ integration limits, respectively. Near $x=0$, it is necessary to perform a Poisson resummation of $p_{a}$ winding numbers. There are two consequences of this operation: appearance of the $\left(V_{p}^{a}\right)^{-1}$ factor which converts the string coupling $g_{s}$ into $g^{2} \equiv g_{a}^{2}$ of the $a$-stack (QCD), see Eq.(5), and exposition of $t$-channel poles associated to the exchanges of massless gluons and of their Kaluza-Klein excitations that can also wind around $\Sigma_{b}$ cycles of the other stack. The masses of these particles are given in Eq. (5.50) of Ref.[6] and can be schematically written as:

$$
M_{b a}^{2}=\left(M_{K K}^{(a)}\right)^{2}+\left(M_{\text {wind. }}^{(b)}\right)^{2}
$$


Note that in the process under consideration, these poles appear outside the physical region of $t \leq 0$, however their effects may compete with the string states if $M_{b a}<M_{s}$. Hence it is important to understand under what circumstances the corresponding contributions to the scattering amplitudes can be neglected. One possibility is to have the QCD KaluzaKlein threshold above the string scale, $V_{p}^{a}<\left(M_{s}\right)^{-p}$, with other cycles being larger, i.e. $V_{p}^{b}>\left(M_{s}\right)^{-p}$. This is certainly consistent with $g_{a}>g_{b}$, i.e., with the QCD coupling being the strongest one. However, it is not possible to accomplish the suppression of all KaluzaKlein particles in this way, for the following reason. Near $x=1$, Poisson resummation in $p_{b}$ brings the volume factor $\left(V_{p}^{b}\right)^{-1}$ which converts the string coupling $g_{s}$ into $g_{b}^{2}$ and exposes the (unphysical) $u$ - channel poles at the masses

$$
M_{a b}^{2}=\left(M_{K K}^{(b)}\right)^{2}+\left(M_{\text {wind. }}^{(a)}\right)^{2},
$$

associated to Kaluza-Klein excitations of electro-weak vector bosons (possibly winding along the QCD cycles). Thus the same volume hierarchy that lifts the QCD KK threshold beyond the string scale brings down the winding states of electro-weak gauge bosons below the string scale. The "consolation prize" is that the corresponding amplitudes come with the smaller electro-weak coupling $g_{b}^{2}$. In general, any hierarchy will leave some KK or winding states below the string scale.

We find that quark-quark scattering amplitudes, which do not exhibit resonant behavior in the physical domain of kinematic variables (except for the massless $t$ - and $u$-channel poles), are particularly sensitive to a possible hierarchy of various compactification cycles because KK and winding modes can produce a considerable "continuum" background below the string threshold. In order to obtain a rough numerical estimate of such effects, we assume $M_{a b}<M_{s}<M_{b a}$ and take the $\alpha^{\prime} \rightarrow 0$ limit of Eq.(23), keeping only the contribution of the lightest excitations of electro-weak bosons:

$$
F_{t u}^{b b}=1+\frac{g_{b}^{2} t}{g_{a}^{2} u}+\frac{g_{b}^{2} t}{g_{a}^{2}} \frac{N_{p} \Delta}{u-M_{a b}^{2}} .
$$

The factor $N_{p}$ takes into account a possible degeneracy of the lowest level while $\Delta \sim e^{-M_{a b}^{2} / M_{s}^{2}}$ takes into account the effects of finite thickness of the brane intersections. Any realistic model must contain D-branes with at least three extra spatial dimensions, therefore it is reasonable to set $N_{p}=3$. D-brane thickness effects are also model-dependent, ${ }^{2}$ but they are exponentially suppressed in the limit $M_{a b} \ll M_{s}$. In our numerical analysis, we will be considering $M_{a b}=0.7 M_{s}$, assumming that such KK scale is sufficiently low for ignoring the effects of D-brane thickness and setting $\Delta=1$. Futhermore, we set

$$
G_{t u}^{b c}=G_{t u}^{\prime b c}=1
$$

\section{String Theory vs. Field Theory $\mapsto U(N)$ vs. $S U(N)$}

As discussed in the Introduction, the gauge group associated with the color and electroweak stacks are $S U(3)_{\mathrm{C}} \times U(1)_{a}$ and $S U(2)_{\mathrm{EW}} \times U(1)_{b}$. These extra $U(1)$ 's are a source

\footnotetext{
${ }^{2}$ In the intersecting brane models discussed in Ref.[6], $\Delta=\delta^{-M_{a b}^{2} / M_{s}^{2}}$, with $\ln \delta=2 \psi(1)-\psi(\theta / \pi)-\psi(1-$ $\theta / \pi)$, where $\theta$ is the angle between electro-weak and QCD stacks. Note that $\delta=16$ for orthogonal stacks.
} 
of dramatic differences in the scattering amplitudes from the field theoretic expectation. A striking example of this can be seen in the $q q^{\prime} \rightarrow q q^{\prime}$ process described by Eqs. (22) and (27). The first term in (27), the "1," describes $t$-channel gluon exchange of an $S U(3)$ gluon, whereas the second term is the contribution from the $u$-channel exchange of a charged $S U(2) W^{ \pm}$. What is apparently missing is the $t$-channel exchange of the neutral $W^{0}$, which is certainly present in the $S U(2)$ field theory.

The source of this effect can be traced to cancelations of the $U(1)_{b}$ and $S U(2)$ contributions. In Ref. [6], a subtle procedure was used for separating $S U(N)$ from $U(1)$, based on the identity

$$
\delta_{\alpha_{2}}^{\alpha_{1}} \delta_{\alpha_{4}}^{\alpha_{3}}=2 \sum_{a}\left(T^{a}\right)_{\alpha_{4}}^{\alpha_{1}}\left(T^{a}\right)_{\alpha_{2}}^{\alpha_{3}}+2 Q_{A}^{2} \delta_{\alpha_{4}}^{\alpha_{1}} \delta_{\alpha_{2}}^{\alpha_{3}}
$$

where the sum is over all $S U(N)$ generators and $Q_{A}=1 / \sqrt{2 N}$ is the properly normalized $U(1)$ charge. This was done for the amplitude involving $S U(2)$ doublets $q_{\beta}^{\alpha}$ labeled by $U(2)$ incides $\beta$ and $U(3)$ (color) indices $\alpha$ :

$$
M\left(q_{\beta_{1}}^{\alpha_{1}} q_{\beta_{2}}^{\alpha_{2}} \rightarrow q_{\beta_{3}}^{\alpha_{3}} q_{\beta_{4}}^{\alpha_{4}}\right) \sim R(t, u) \delta^{\alpha_{1} \alpha_{4}} \delta^{\alpha_{2} \alpha_{3}} \times \delta^{\beta_{1} \beta_{3}} \delta^{\beta_{2} \beta_{4}}+R(u, t) \delta^{\alpha_{1} \alpha_{3}} \delta^{\alpha_{2} \alpha_{4}} \times \delta^{\beta_{1} \beta_{4}} \delta^{\beta_{2} \beta_{3}}
$$

where

$$
R(t, u)=\frac{g_{a}^{2}}{t}+\frac{g_{b}^{2}}{u}+\ldots
$$

Now consider the process $u^{\alpha_{1}} d^{\alpha_{2}} \rightarrow u^{\alpha_{3}} d^{\alpha_{4}}$. According to the string-theoretical Eq.(30), only the first term contributes in this case. There is a gluon pole in the $t$-channel and an electro-weak (charged $W$ ) pole in the $u$-channel, captured by the $F_{t u}$ term in (22). So what has happened to the $W^{0}$ exchange in the $t$-channel? A priori, it is contained in the second term of Eq.(30). Indeed, by using Eq.(29), its electro-weak $U(2)_{b}$ group factor can be rewritten as:

$$
\delta^{\beta_{1} \beta_{4}} \delta^{\beta_{2} \beta_{3}}=2\left(T^{1}\right)^{\beta_{1} \beta_{3}}\left(T^{1}\right)^{\beta_{2} \beta_{4}}+2\left(T^{2}\right)^{\beta_{1} \beta_{3}}\left(T^{2}\right)^{\beta_{2} \beta_{4}}+2\left(T^{3}\right)^{\beta_{1} \beta_{3}}\left(T^{3}\right)^{\beta_{2} \beta_{4}}+\frac{1}{2} I^{\beta_{1} \beta_{3}} I^{\beta_{2} \beta_{4}},
$$

with the third term on the r.h.s. representing $W^{0}$ exchange between $u$ and $d$ quarks. Furthermore, the kinematic factor $R(u, t)$ supplies the corresponding $g_{b}^{2} / t$ pole term. However, as it is made explicit in Eq.(32), this $W^{0}$ contribution is canceled by the contribution of the extra $Z^{\prime}$ gauge boson associated to $U(1)_{b}$. Note that $U(1)_{b}$ is anomalous and, in the context of realistic models, it combines with other anomalous $U(1)$ 's to anomaly-free hypercharge $U(1)_{Y}$ and a number of other $U(1)$ 's coupled to anomalous currents. At the end of the day, the corresponding $Z^{\prime}$-bosons become massive [15]. This suggests removing "by hand" the last term from the r.h.s. of Eq.(32), which leaves $W^{0}$ as the only (massless) neutral particle exchanged in the $t$-channel. (Because of the flavor assignments for $u d \rightarrow u d$, the first two terms in Eq.(32) are zero.) However, such an ad hoc procedure introduces its own complications in the form of a QCD-strength $\left(\sim g_{a}^{2} / u\right)$ pole in the $u$-channel, because this unwanted term cannot be separated from the desired $t$-channel pole if one wants to preserve the $t$ - $u$ channel string duality of $R(u, t)$. In the following section we will address phenomenological approaches to extract information on these interesting aspects of four-fermion amplitudes.

\section{DIJET ANGULAR DISTRIBUTIONS}

QCD parton-parton cross sections are dominated by $t$-channel exchanges that produce dijet angular distributions which peak at small center of mass scattering angles, $\theta^{*}$. In 
contrast, non-standard contact interactions or excitations of resonances result in a more isotropic distribution. In terms of rapidity variable for standard transverse momentum cuts, dijets resulting from QCD processes will preferentially populate the large rapidity region, while the new processes generate events more uniformly distributed in the entire rapidity region. To analyze the details of the rapidity space the $\mathrm{D} \varnothing$ Collaboration introduced a new parameter [16],

$$
R=\frac{d \sigma /\left.d M\right|_{\left(\left|y_{1}\right|,\left|y_{2}\right|<0.5\right)}}{d \sigma /\left.d M\right|_{\left(0.5<\left|y_{1}\right|,\left|y_{2}\right|<1.0\right)}},
$$

the ratio of the number of events, in a given dijet mass bin, for both rapidities $\left|y_{1}\right|,\left|y_{2}\right|<0.5$ and both rapidities $0.5<\left|y_{1}\right|,\left|y_{2}\right|<1.0$. Here, $M$ is the dijet invariant mass. With the definitions $Y \equiv \frac{1}{2}\left(y_{1}+y_{2}\right)$ and $y \equiv \frac{1}{2}\left(y_{1}-y_{2}\right)$, the cross section per interval of $M$ for $p p \rightarrow$ dijet is given by

$$
\begin{aligned}
\frac{d \sigma}{d M} & =M \tau \sum_{i j k l}\left[\left.\int_{-Y_{\max }}^{0} d Y f_{i}\left(x_{a}, M\right) f_{j}\left(x_{b}, M\right) \int_{-\left(y_{\max }+Y\right)}^{y_{\max }+Y} d y \frac{d \sigma}{d \widehat{t}}\right|_{i j \rightarrow k l} \frac{1}{\cosh ^{2} y}\right. \\
& \left.+\left.\int_{0}^{Y_{\max }} d Y f_{i}\left(x_{a}, M\right) f_{j}\left(x_{b}, M\right) \int_{-\left(y_{\max }-Y\right)}^{y_{\max }-Y} d y \frac{d \sigma}{d \widehat{t}}\right|_{i j \rightarrow k l} \frac{1}{\cosh ^{2} y}\right]
\end{aligned}
$$

where $\tau=M^{2} / s, x_{a}=\sqrt{\tau} e^{Y}, x_{b}=\sqrt{\tau} e^{-Y}$, and

$$
|\mathcal{M}(i j \rightarrow k l)|^{2}=\left.16 \pi \widehat{s}^{2} \frac{d \sigma}{d \widehat{t}}\right|_{i j \rightarrow k l}
$$

In this section we reinstate the caret notation $(\widehat{s}, \widehat{t}, \widehat{u})$ to specify partonic processes. The $Y$ integration range in Eq. (34), $Y_{\max }=\min \left\{\ln (1 / \sqrt{\tau}), y_{\max }\right\}$, comes from requiring $x_{a}, x_{b}<1$ together with the rapidity cuts $y_{\min }<\left|y_{1}\right|,\left|y_{2}\right|<y_{\max }$. The kinematics of the scattering also provides the relation $M=2 p_{T} \cosh y$, which when combined with $p_{T}=M / 2 \sin \theta^{*}=$ $M / 2 \sqrt{1-\cos ^{2} \theta^{*}}$, yields $\cosh y=\left(1-\cos ^{2} \theta^{*}\right)^{-1 / 2}$. Finally, the Mandelstam invariants occurring in the cross section are given by $\widehat{s}=M^{2}, \widehat{t}=-\frac{1}{2} M^{2} e^{-y} / \cosh y$, and $\widehat{u}=$ $-\frac{1}{2} M^{2} e^{+y} / \cosh y$. The ratio $R$ is a genuine measure of the most sensitive part of the angular distribution, providing a single number that can be measure as a function of the dijet invariant mass [17].

In assessing the four-fermion amplitudes there are two independent contributions of stringy physics: (i) the presence of KK and winding modes; (ii) anomalous effects in $q q^{\prime} \rightarrow q q^{\prime}$ because of cancelations between $U(1)$ and $S U(N)$ contributions. The latter acquires complexity because the unknown anomalous masses of the $U(1)$ gauge bosons disturb this cancelation. In order to simplify the analysis we present two extreme cases. Firstly, we include a single KK contribution in $q q \rightarrow q q$, while omitting the non-QCD part of $q q^{\prime} \rightarrow q q^{\prime}$ which contains the anomalous $U(1)$ cancelation. The importance of this omission is somewhat mitigated because the $q q^{\prime}$ valence quark luminosity is only $4 / 5$ of the $q q$ luminosity. Secondly, we go to the opposite extreme case by omitting the KK contribution and including the $q q^{\prime} \rightarrow q q^{\prime}$ amplitude in the limit where all the gauge bosons are massless.

In Figs. 1 and 2 we compare the results from a full CMS detector simulation of the ratio $R$, with predictions from LO QCD, model-independent contributions to the $q^{*}, g^{*}$ and $C^{*}$ excitations, and the non-resonant string scattering. The synthetic population was generated with Pythia, passed through the full CMS detector simulation and reconstructed with the 

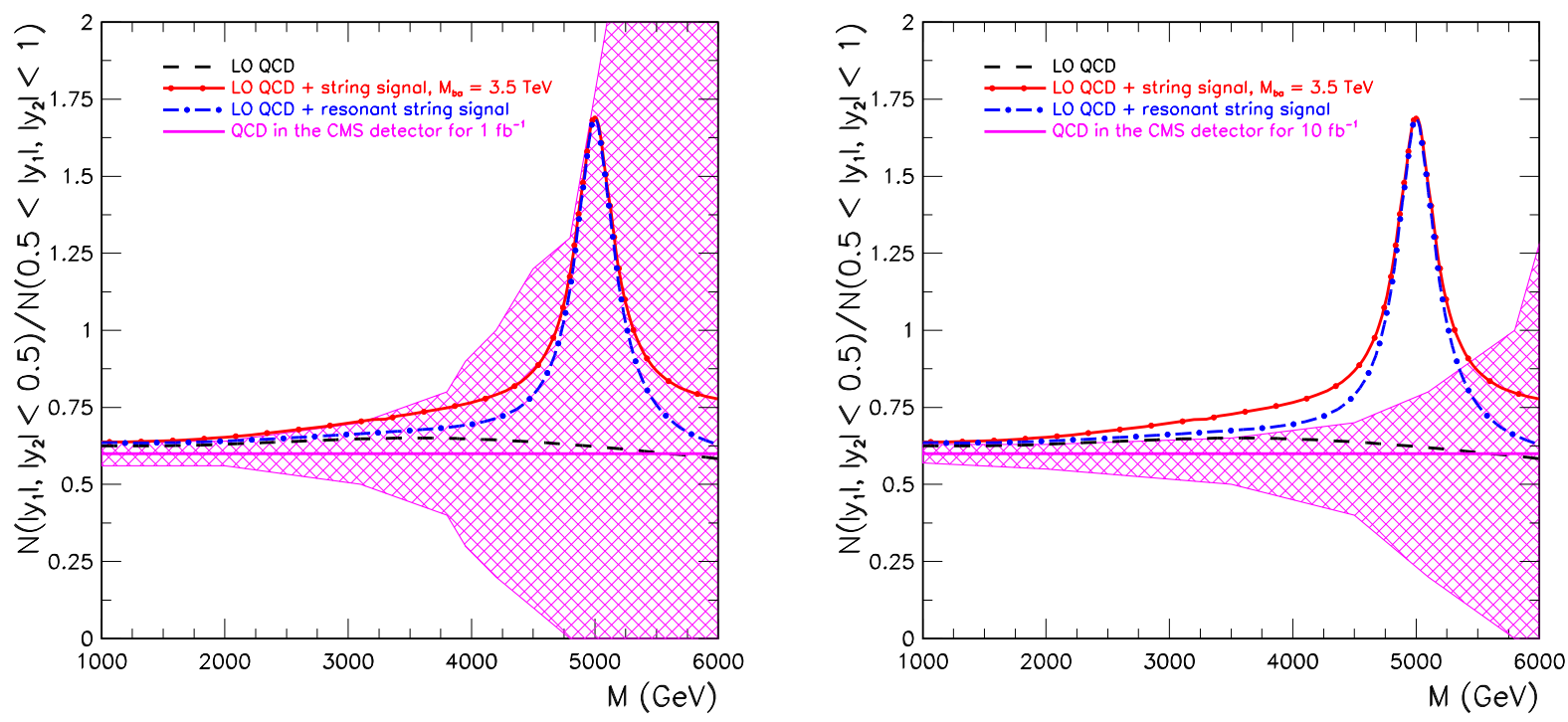

FIG. 1: For integrated luminosities of $1 \mathrm{fb}^{-1}$ (left panel) and $10 \mathrm{fb}^{-1}$ (right panel) the expected value (solid line) and statistical error (shaded region) of the dijet ratio of QCD in the CMS detector [19] is compared with LO QCD (dashed line), LO QCD + lowest massive string excitations (dot-dashed), and LO QCD + lowest massive string excitations + non-resonant string scattering in $q q \rightarrow q q$ (dot-solid). We have taken $M_{a b}=3.5 \mathrm{TeV}, M_{s}=5 \mathrm{TeV}$, and $g_{b}^{2} / g_{a}^{2}=1 / 3$.

ORCA reconstruction package [19]. For an integrated luminosity of $10 \mathrm{fb}^{-1}$ the LO QCD contributions with $\alpha_{\mathrm{QCD}}=0.1$ (corresponding to running scale $\mu \approx M_{s}$ ) are within statistical fluctuations of the full CMS detector simulation. Since one of the purposes of utilizing NLO calculations is to fix the choice of the running coupling, we take this agreement as rationale to omit loops in QCD and in string theory. It is clear from Fig. 1 that incorporating NLO calculation of the background and the signal would not significantly change the deviation of the string contribution from the QCD background.

In Fig. 1 we show the contribution of the lightest KK mode (in the isotropic case with lowest KK level degeneration $N_{p}=3$ ) for $M_{a b}=3.5 \mathrm{TeV}$. In Fig. 2 we show the anomalous effect in $q q^{\prime} \rightarrow q q^{\prime}$ amplitude in the limit where all the gauge bosons are massless. We note that inclusion of anomalous masses reinstitute the $W^{0}$ pole in the $q q^{\prime}$ amplitude and this has the effect of decreasing the deviation of $R$ from its QCD behavior. In that case we expect the deviation to be dominated by the finite mass KK contribution, shown in Fig. 1. In order to assess the statistical significance of this claim, we calculate the signal-to-noise $(S / \sqrt{B})$ ratio for events with both rapidities between $(0,0.5)$ and invariant mass in the interval $3 \mathrm{TeV}<M<3.5 \mathrm{TeV}$. For $10 \mathrm{fb}^{-1}$ we find the following contributions with respect to the SM background $(B)$ : (i) from the tail of the Regge excitation $S / \sqrt{B}=100 / 48=2 \sigma$; (ii) from the KK modes $S / \sqrt{B}=290 / 48=6 \sigma$. It is important to emphasize that the KK contribution viewed as a contact term is prescribed by the theory, in the form, the sign, and strength relative to the QCD and Regge contributions. 

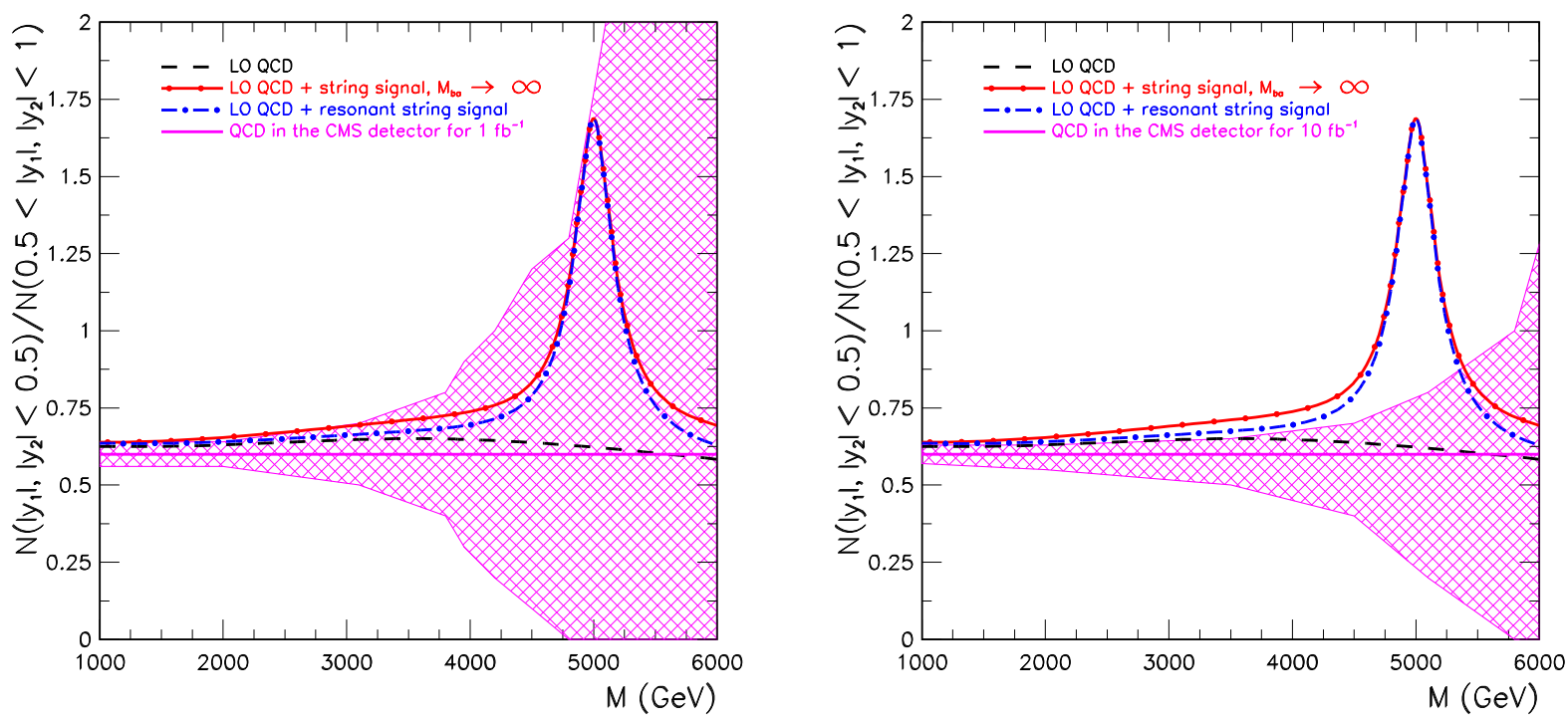

FIG. 2: For integrated luminosities of $1 \mathrm{fb}^{-1}$ (left panel) and $10 \mathrm{fb}^{-1}$ (right panel) the expected value (solid line) and statistical error (shaded region) of the dijet ratio of QCD in the CMS detector [19] is compared with LO QCD (dashed line), LO QCD + lowest massive string excitations (dot-dashed), and LO QCD + lowest massive string excitations + non-resonant string scattering in $q q \rightarrow q q$ and $q q^{\prime} \rightarrow q q^{\prime}$ (dot-solid), with all anomalous $U(1)$ masses set to zero (see text). We have taken $M_{a b}=\infty \mathrm{TeV}, M_{s}=5 \mathrm{TeV}$, and $g_{b}^{2} / g_{a}^{2}=1 / 3$.

\section{MONOJET, TRIJET, AND JET PLUS DILEPTON CONFIGURATIONS}

Events with a single jet plus missing energy $\left(\mathbb{E}_{T}\right)$ with balancing transverse momenta (so-called "monojets") are incisive probes of new physics, and therefore enormous effort has been dedicated to identify the underlying model which can produce them [20]. In order to optimize our chances of uncovering low mass string excitations, in what follows we analyze the predictions of D-brane compactifications in the monojet channel.

As in the SM, the source of this topology is $i j \rightarrow k Z^{0}$ followed by $Z^{0} \rightarrow \nu \bar{\nu}$. Both in the SM and string theory the cross section for this process is of order $g^{4}$. Virtual KK graviton emission $(i j \rightarrow k G)$ involves emission of closed strings, resulting in an additional suppression of order $g^{2}$ compared to $Z^{0}$ emission. A careful discussion of this suppression is given in Ref. [8]. However, in some scenarios compensation for this suppression can arise from the large multiplicity of graviton emission, which is somewhat dependent on the cutoff mechanism [21]. Additional evidence for string excitations can be obtained through analysis of the related process $Z^{0} \rightarrow l^{+} l^{-}$. For $l=e, \mu$ the final topology is jet + dilepton. For $l=\tau$, about two-thirds of the subsequent tau decays are hadronic and so in $45 \%$ of cases the observed configuration is a trijet.

As in the dijet analysis we consider scattering processes on the (color) $U(3)_{a}$ stack of D-branes, leading now to $Z^{0}+$ jet final state. Recall that the $U(1)_{Y}$ boson $Y_{\mu}$, which gauges the usual electroweak hypercharge symmetry, is a linear combination of $C_{\mu}$, the $U(1)_{c}$ boson $B_{\mu}$ terminating on a separate brane, and perhaps a third additional $U(1)_{b}$ (say $\left.W_{\mu}\right)$ sharing a $U(2)_{b}$ brane which is also a terminus for the $S U(2)_{L}$ electroweak gauge bosons $W_{\mu}^{a}$. Thus, critically for our purposes, the $Z_{\mu}$ boson, which is a linear combination of $Y_{\mu}$ and $W_{\mu}^{3}$ will 
TABLE I: Signal-to-noise ratio $(S / \sqrt{B})$ of the jet $+\mathscr{E}_{T}$, jet + dilepton, and trijet channels for an integrated luminosity of $100 \mathrm{fb}^{-1}$.

\begin{tabular}{c|c|c|c}
\hline \hline$M_{s}(\mathrm{TeV})$ & jet $+\mathscr{E}_{T}$ & jet + dilepton & trijet \\
\hline 1 & $21181 / 135$ & $6989 / 78$ & $1553 / 37$ \\
2 & $719 / 31$ & $273 / 18$ & $53 / 9$ \\
3 & $69 / 11$ & $23 / 6$ & $5 / 3$ \\
4 & $9 / 4$ & $3 / 2$ & $1 / 1$ \\
\hline \hline
\end{tabular}

participate with the gluon octet in (string) tree level scattering processes on the color brane, processes which in the SM occur only at one-loop level. Such a mixing between hypercharge and baryon number is a generic property of D-brane quivers, see e.g. Refs. [22-24].

By duplicating the discussion in Ref. [3] we obtain the $s$-channel pole terms of the average square amplitudes contributing to $p p \rightarrow Z^{0}+$ jet. The dominant terms are

$$
|\mathcal{M}(g g \rightarrow g Z)|^{2}=\frac{5}{3} Q^{2} \frac{g^{4}}{M_{s}^{4}}\left[\frac{M_{s}^{8}}{\left(s-M_{s}^{2}\right)^{2}+\left(\Gamma_{g^{*}}^{J=0} M_{s}\right)^{2}}+\frac{t^{4}+u^{4}}{\left(s-M_{s}^{2}\right)^{2}+\left(\Gamma_{g^{*}}^{J=2} M_{s}\right)^{2}}\right]
$$

and

$$
|\mathcal{M}(q g \rightarrow q Z)|^{2}=-\frac{1}{3} Q^{2} \frac{g^{4}}{M_{s}^{2}}\left[\frac{M_{s}^{4} u}{\left(s-M_{s}^{2}\right)^{2}+\left(\Gamma_{q^{*}}^{J=\frac{1}{2}} M_{s}\right)^{2}}+\frac{u^{3}}{\left(s-M_{s}^{2}\right)^{2}+\left(\Gamma_{q^{*}}^{J=\frac{3}{2}} M_{s}\right)^{2}}\right]
$$

where

$$
Q=\sqrt{\frac{1}{6}} \kappa \sin \theta_{W} \approx 2.76 \times 10^{-2}\left(\frac{\kappa}{0.14}\right)
$$

is the product of the $U(1)$ charge of the fundamental representation $(\sqrt{1 / 6})$ followed by successive projections onto the hypercharge $(\kappa)$ and then onto the $Z^{0}$-boson $\left(\sin \theta_{W}\right)$. In a self evident notation the $\Gamma_{\left(q^{*}, g^{*}\right)}^{J}$ 's are the total widths of the participating resonances. These are calculated in Ref. [5].

By convoluting with parton distribution functions we calculate $d \sigma / d M$, where $M$ is the invariant mass of $Z^{0}+$ jet [25]. We then multiply by the corresponding branching ratio $\left(Z^{0} \rightarrow \nu \bar{\nu}, Z^{0} \rightarrow e^{+} e^{-}+\mu^{+} \mu^{-}, Z^{0} \rightarrow \tau^{+} \tau^{-}\right)$and integrate over the region $M_{s}-2 \Gamma_{\left(q^{*}, g^{*}\right)}^{J}<$ $M<M_{s}+2 \Gamma_{\left(q^{*}, g^{*}\right)}^{J}$ (with the appropriate width for each partonic subprocess) to obtain the resonant cross section for the different topologies. For an integrated luminosity of $100 \mathrm{fb}^{-1}$ one arrives at the signal-to-noise ratio given in Table I. The noise indicates the square root of the QCD background number of events in each of these channels (for obtaining the background in the resonant region we integrate over the interval $M_{s}-2 \Gamma_{\max }<M<$ $M_{s}+2 \Gamma_{\max }$, where $\Gamma_{\max }$ is the largest width).

From Table I we see that the LHC reach for identifying stringy contributions from $p p \rightarrow$ $Z^{0}+$ jet is about $M_{s}=3.5 \mathrm{TeV}$, based on monojets and jet + dilepton events. We can compare this with the sensitivity previously obtained for $p p \rightarrow \operatorname{direct} \gamma+$ jet $\left(M_{s}=5 \mathrm{TeV}\right)$ and in the dijet channel $\left(M_{s}=6.8 \mathrm{TeV}\right.$, with $\left.S / \sqrt{B}=210 / 42\right)[7]$. Thus, if $M_{s} \lesssim 3.5 \mathrm{TeV}$, the $Z^{0}$ channel can serve as significant corroborative evidence for stringy contributions, with the dijet channel clearly best suited for discovery. 


\section{LHC SENSITIVITY FOR EARLY DISCOVERY OF STRING RESONANCES}

The LHC is expected soon to begin circulation of beams for production of $p p$ collisions at $\sqrt{s}=10 \mathrm{TeV}$, followed by a physics run at $\sqrt{s}=14 \mathrm{TeV}$. In the LHC ramping up process it will be crucial to observe various familiar SM processes at their expected rates, with distributions and mass peaks at previously measured values. Conventional wisdom holds that once confidence in the ATLAS and CMS detectors has been deep-rooted, the search for beyond SM physics will start. Of course, new exotic phenomena could have cross sections so large, and topologies so striking, that even a limited amount of collected data and a non-ultimate detector performance could lead to exciting results. In the spirit of [26], in this section we explore the possibility of searching for lowest massive Regge excitations of open strings in parallel with the calibration phase. We will show that even with relatively poor knowledge of detector response, searches of Regge recurrences could still be possible.

Following our previous analysis [7], we study the discovery reach of Regge excitations in data binned according to the invariant mass $M$ of the dijet, after setting cuts on the different jet rapidities, $\left|y_{1}\right|,\left|y_{2}\right| \leq 1$ [27] and transverse momenta $p_{\mathrm{T}}^{1,2}>50 \mathrm{GeV}$. In Fig. 3 we show representative plots of the invariant mass spectrum, for $M_{s}=2 \mathrm{TeV}$ and $M_{s}=3 \mathrm{TeV}$. The string signal has been calculated using (34) with the corresponding $2 \rightarrow 2$ scattering amplitudes given in Eqs. (13) - (16). The QCD background has been calculated at the partonic level from the same processes as designated for the signal, with the addition of $q q \rightarrow q q$ and $q \bar{q} \rightarrow q \bar{q}$.

We now estimate (at the parton level) the early LHC discovery reach. Standard bumphunting methods, such as obtaining cumulative cross sections, $\sigma\left(M_{0}\right)=\int_{M_{0}}^{\infty} \frac{d \sigma}{d M} d M$, from the data and searching for regions with significant deviations from the QCD background, may reveal an interval of $M$ suspected of containing a bump. With the establishment of such a region, one may calculate a signal-to-noise ratio with the signal rate once more estimated in the invariant mass window $\left[M_{s}-2 \Gamma, M_{s}+2 \Gamma\right]$, and $\sqrt{B}$ taken in the same dijet mass interval for the same integrated luminosity.

For $M_{s}=3 \mathrm{TeV}$ and $100 \mathrm{pb}^{-1}$ of data collected at $\sqrt{s}=10 \mathrm{TeV}$, we expect $S / \sqrt{B}=$ $127 / 20=6 \sigma$. For an overly conservative assumption of integrated luminosity $\approx 10 \mathrm{pb}^{-1}$, a $S / \sqrt{B}=204 / 19>10 \sigma$ is expected for string scales as high as $M_{s}=2 \mathrm{TeV}$. It is remarkable that within 1 year of data collection at $\sqrt{s}=10 \mathrm{TeV}$, string scales as large as $3 \mathrm{TeV}$ are open to discovery at the $\geq 5 \sigma$ level. Once more, we stress that these results contain no unknown parameters. They depend only on the D-brane construct for the SM, and are independent of compactification details.

\section{CONCLUSIONS}

- In the framework of D-branes, we have reviewed the theoretical complexity of fourfermion scattering amplitudes in low scale string theory, focussing on the aspects which are central to a phenomenological analysis. These consist of a significant interplay among string Regge recurrences, Kaluza-Klein modes, and D-brane winding modes. The relative contributions of KK and winding modes also reflect the irreducible correlation between strong and electroweak interactions inherent in the intersecting brane picture.

- Although there are no $s$-channel resonances in $q q \rightarrow q q$ and $q q^{\prime} \rightarrow q q^{\prime}$ scattering, KK 


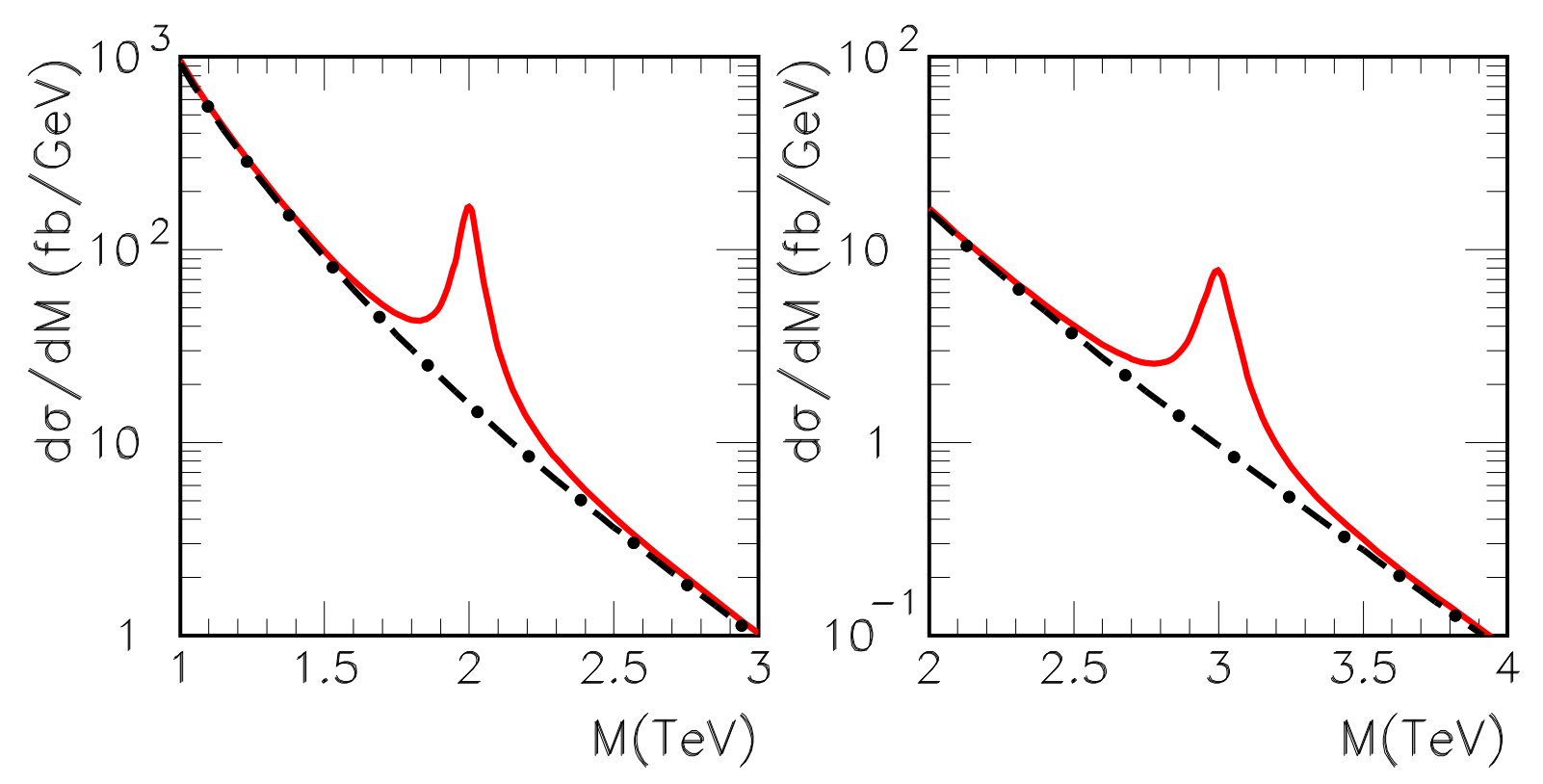

FIG. 3: $d \sigma / d M$ (units of $\mathrm{fb} / \mathrm{GeV}$ ) vs. $M(\mathrm{TeV}$ ) is plotted for the case of SM QCD background (dot-dashed line) and (first resonance) string signal + background (solid line), for $M_{s}=2 \mathrm{TeV}$ (left) and $M_{s}=3 \mathrm{TeV}$ (right).

modes in the $t$ and $u$ channels generate calculable effective 4 -fermion contact terms. These in turn are manifest in an enhancement in the continuum below the string scale of the $R$ ratio for dijet events, described in the text. For $M_{\mathrm{KK}} \leq 3 \mathrm{TeV}$, this contribution can be detected at the LHC with $6 \sigma$ significance above SM background. In combination with the simultaneous observation in dijet events of a string resonance at $M_{s}>M_{\mathrm{KK}}$, this would consolidate the stringy interpretation of these anomalies.

- Analyses of the various final-state topologies arising in the $p p \rightarrow Z^{0}+$ jet channel show that, for $M_{s}<3.5 \mathrm{TeV}$, monojet, trijet, and dilepton plus jet configurations could provide corroborative evidence for Regge excitations in D-brane models.

- Excitation cross sections for Regge recurrences in the early LHC run, operating at $5 \mathrm{TeV}$ per beam are sizeable. Consequently, dijet signals of string excitations may be so large for $M_{s} \lesssim 3 \mathrm{TeV}$ that they could be detected at statistically significant event rates in the first year of running.

\section{Acknowledgments}

We are grateful to Lance Dixon for continuous inspiring discussions. L.A.A. is supported by the U.S. National Science Foundation Grant No PHY-0757598, and the UWM Research Growth Initiative. H.G. is supported by the U.S. National Science Foundation Grant No PHY-0757959. The research of D.L. and St.St. is supported in part by the European Commission under Project MRTN-CT-2004-005104. S.N. is supported by the UWM Research Growth Initiative. He is grateful to Max-Planck-Institut für Physik in München, for their kind hospitality. The research of T.R.T. is supported by the U.S. National Science Foun- 
dation Grants PHY-0600304, PHY-0757959 and by the Cluster of Excellence "Origin and Structure of the Universe" in München, Germany. Any opinions, findings, and conclusions or recommendations expressed in this material are those of the authors and do not necessarily reflect the views of the National Science Foundation.

[1] N. Arkani-Hamed, S. Dimopoulos and G. R. Dvali, Phys. Lett. B 429, 263 (1998) [arXiv:hep$\mathrm{ph} / 9803315]$.

[2] I. Antoniadis, N. Arkani-Hamed, S. Dimopoulos and G.R. Dvali, Phys. Lett. B 436, 257 (1998) [arXiv:hep-ph/9804398].

[3] L. A. Anchordoqui, H. Goldberg, S. Nawata and T.R. Taylor, Phys. Rev. Lett. 100, 171603 (2008) [arXiv:0712.0386 [hep-ph]].

[4] L. A. Anchordoqui, H. Goldberg, S. Nawata and T.R. Taylor, Phys. Rev. D 78, 016005 (2008) [arXiv:0804.2013 [hep-ph]].

[5] L. A. Anchordoqui, H. Goldberg and T.R. Taylor, Phys. Lett. B 668, 373 (2008) [arXiv:0806.3420 [hep-ph]].

[6] D. Lüst, S. Stieberger and T.R. Taylor, Nucl. Phys. B 808, 1 (2009) [arXiv:0807.3333 [hep-th]].

[7] L.A. Anchordoqui, H. Goldberg, D. Lüst, S. Nawata, S. Stieberger and T.R. Taylor, Phys. Rev. Lett. 101, 241803 (2008) [arXiv:0808.0497 [hep-ph]].

[8] S. Cullen, M. Perelstein and M. E. Peskin, Phys. Rev. D 62, 055012 (2000) [arXiv:hep$\mathrm{ph} / 0001166]$.

[9] E. Accomando, I. Antoniadis and K. Benakli, Nucl. Phys. B 579, 3 (2000) [arXiv:hep$\mathrm{ph} / 9912287]$.

[10] E. Dudas and J. Mourad, Nucl. Phys. B 575, 3 (2000) [arXiv:hep-th/9911019].

[11] D. Chialva, R. Iengo and J.G. Russo, Phys. Rev. D 71, 106009 (2005) [arXiv:hep-ph/0503125].

[12] For a review, see: R. Blumenhagen, B. Körs, D. Lüst and S. Stieberger, Phys. Rept. 445, 1 (2007) [arXiv:hep-th/0610327].

[13] G. Veneziano, Nouvo Cimento A 57, 190 (1968).

[14] Aspects of four fermion scattering in intersecting D6 brane models have been discussed by M. Chemtob, Phys. Rev. D 78, 125020 (2008) [arXiv:0808.1242 [hep-ph]].

[15] D. M. Ghilencea, L. E. Ibanez, N. Irges and F. Quevedo, JHEP 0208, 016 (2002) [arXiv:hep$\mathrm{ph} / 0205083]$.

[16] B. Abbott et al. [D0 Collaboration], Phys. Rev. Lett. 82, 2457 (1999) [arXiv:hep-ex/9807014].

[17] An illustration of the use of this parameter in a heuristic model where standard model amplitudes are modified by a Veneziano formfactor has been presented by P. Meade and L. Randall, JHEP 0805, 003 (2008) [arXiv:0708.3017 [hep-ph]].

[18] J. Pumplin, D. R. Stump, J. Huston, H. L. Lai, P. Nadolsky and W. K. Tung, JHEP 0207, 012 (2002) [arXiv:hep-ph/0201195].

[19] S. Esen and R. Harris, CMS Note 2006/071.

[20] See e.g., T. G. Rizzo, Phys. Lett. B 665, 361 (2008) [arXiv:0805.0281 [hep-ph]].

[21] M. Bando, T. Kugo, T. Noguchi and K. Yoshioka, Phys. Rev. Lett. 83, 3601 (1999) [arXiv:hepph/9906549]. L. A. Anchordoqui, J. L. Feng, H. Goldberg and A. D. Shapere, Phys. Rev. D 65, 124027 (2002) [arXiv:hep-ph/0112247]; J. Hewett and T. Rizzo, JHEP 0712, 009 (2007) [arXiv:0707.3182 [hep-ph]].

[22] I. Antoniadis, E. Kiritsis and T. N. Tomaras, Phys. Lett. B 486, 186 (2000) [arXiv:hep- 
$\mathrm{ph} / 0004214]$.

[23] R. Blumenhagen, B. Körs, D. Lüst and T. Ott, Nucl. Phys. B 616, 3 (2001) [arXiv:hepth/0107138].

[24] D. Berenstein and S. Pinansky, Phys. Rev. D 75, 095009 (2007) [arXiv:hep-th/0610104].

[25] Monte Carlo simulations interfaced with PYTHIA has been carried out in this process by A. Roy and M. Cavaglia, arXiv:0902.1473 [hep-ph]. Their results are in relatively good agreement with the parton level calculation in the present work.

[26] F. Gianotti and M. L. Mangano, arXiv:hep-ph/0504221; H. Baer, V. Barger and G. Shaughnessy, Phys. Rev. D 78, 095009 (2008) [arXiv:0806.3745 [hep-ph]]; M. L. Mangano, Eur. Phys. J. C 59, 373 (2009) [arXiv:0809.1567 [hep-ph]]; H. Baer, A. Lessa and H. Summy, arXiv:0809.4719 [hep-ph].

[27] A. Bhatti et al., arXiv:0807.4961 [hep-ex]. 